\title{
Emerging tropical neurological infections
}

\author{
Author: Udaya K Ranawaka ${ }^{\mathrm{A}}$
}

\begin{abstract}
A large majority of neurological infections remain undiagnosed worldwide. Emerging and re-emerging infections are likely to be responsible for a significant proportion of these. Over the last two decades, several new organisms producing neurological infection and the neurotropic potential of many other known pathogens have been identified. Large outbreaks caused by re-emerging pathogens such as Chikungunya virus, Zika virus and Ebola virus have led to better delineation of their neurological manifestations. Recognition of the pandemic potential of emerging pathogens and an improved understanding of their host-vector-environment interactions would help us be better prepared to meet these emerging threats.
\end{abstract}

\section{Introduction}

The global landscape of neurological infections appears to be changing, and a key reason is the emergence and re-emergence of many neurotropic pathogens. This is especially true in tropical countries where the pattern of infectious diseases is different from temperate regions due to a multitude of geographical, socio-economic and socio-cultural factors. Aetiological agents traditionally taught in medical schools as common causes of neurological infections are seldom identified in outbreak investigations and surveillance studies from tropical countries. ${ }^{1,2}$ This article will focus on some emerging neurological infections that have recently gained global attention.

\section{Dengue with neurological involvement}

Dengue is the most important arboviral disease worldwide and is endemic in more than 100 countries, with half of the world population at risk. ${ }^{3}$ Dengue virus (DENV, family Flaviviridae, genus Flavivirus, transmitted by Aedes mosquitoes) has four antigenically distinct serotypes (DENV-1 to DENV-4). Neurological involvement is seen in up to $21 \%$ of patients and is mainly associated with DENV-2 and DENV-3 serotypes. ${ }^{4,5}$ DENV is identified in $4-47 \%$ of suspected encephalitis cases in endemic areas. ${ }^{4} \mathrm{~A}$ high index of suspicion is required as many patients with neurological involvement do not exhibit features of the typical dengue fever syndrome. ${ }^{5}$

The spectrum of neurological manifestations in dengue is diverse. Dengue encephalitis and encephalopathy present with

Author: ${ }^{\text {A }}$ professor in neurology, University of Kelaniya, Kelaniya, Sri Lanka altered consciousness, behavioural changes, headache, seizures and focal deficits. Encephalopathy is the commonest neurological abnormality reported, and results from several systemic disturbances (prolonged shock, hypoxia, metabolic derangements, intracerebral haemorrhage, brain oedema, acute liver failure or renal failure). Cerebrospinal fluid (CSF) analysis is usually normal. Neuroinvasive and neurotropic properties of DENV have been demonstrated over the recent years, confirming the existence of dengue encephalitis. Its diagnosis is supported by lymphocytic pleocytosis, detection of dengue-specific IgM, NS1 antigen or dengue-RNA (by RT-PCR), or virus isolation in CSF. Brain MRI may be normal or show cerebral oedema or non-specific changes. DENV antigen and RNA (by RT-PCR) have been detected from brain tissue in autopsy studies. ${ }^{4,5}$

Dengue-associated muscle dysfunction (myalgia, proximal weakness, elevated creatine kinase), while usually self-limiting, may occasionally lead to rhabdomyolysis. Typical myopathic changes are not usually seen on electromyography. Stroke following dengue is rare, and haemorrhagic strokes are commoner

\section{Key points}

The global landscape of central nervous system infections is rapidly changing.

Neurological syndromes caused by many emerging pathogens, mainly viruses, have been defined in the last decade.

Emerging infections are likely to be responsible for a significant proportion of the burden of neurological infections, especially in tropical countries.

Outbreaks caused by emerging pathogens may result from the expansion of their geographical range, spread from animal reservoirs and development of new neurovirulence.

Diagnostic yields will improve if we start searching for emerging pathogens in observational studies, outbreak investigations and surveillance studies of neurological infections.

KEYWORDS: neurological infections, emerging infections, tropical infections

DOI: 10.7861/clinmed.2021-0799 
than infarcts. Post-infectious immune mediated phenomena include acute disseminated encephalomyelitis (ADEM), GuillainBarré syndrome (GBS), cerebellitis, transverse myelitis, optic neuritis and cranial or peripheral mononeuropathies. ${ }^{5}$

\section{Nipah virus encephalitis}

Nipah virus (NiV, family Paramyxoviridae, genus Henipavirus) was isolated during an outbreak of zoonotic encephalitis in the Nipah valley, Malaysia in 1998, and deadly outbreaks were later reported in South and Southeast Asia. ${ }^{6-9}$ Fruit bats or flying foxes (Pteropus spp.) are natural reservoir hosts. Pigs are infected by direct exposure to bat urine or consumption of contaminated fruits, and act as intermediate amplifier hosts. Humans are affected by contact with infected pigs, direct transmission from bats (by drinking date palm sap contaminated with bat urine and saliva), or human-to-human transmission. ${ }^{6-9}$ The illness is similar to Hendravirus infections in Australia. ${ }^{8,9}$

Asymptomatic infection is uncommon. The illness starts as a typical viral prodrome, and can lead to severe neurological or pulmonary involvement. NiV encephalitis manifests as altered consciousness progressing rapidly to coma, seizures, myoclonus, areflexic weakness, meningism, cerebellar ataxia, and brainstem involvement with autonomic dysregulation. ${ }^{7-9}$ Delayed onset or relapsing encephalitis can occur after months or years, possibly related to reactivation of latent CNS infection. ${ }^{7,9}$ A systemic vasculitis with thrombosis and tissue necrosis, especially affecting the CNS, is noted on autopsy, and vasculitis-related subcortical microinfarcts are seen on brain MRI. ${ }^{7,9}$ Lymphocytic pleocytosis in CSF and diffuse slowing or periodic complexes on EEG are commonly seen. Diagnosis is based on detection of IgM or IgG antibodies in serum. Case fatality is over $70 \% .^{7-9}$

\section{Chikungunya with neurological involvement}

Chikungunya virus (CHIKV, family Togaviridae, genus Alphavirus, transmitted by Aedes mosquitoes) was first isolated in Tanzania in 1953, but gained international attention following the explosive 2005-2007 outbreaks in the Indian Ocean islands. ${ }^{6,710}$ Efficient transmission is maintained without animal reservoirs as infected individuals develop high levels of viraemia, infect mosquitoes and act as reservoirs for infection (similar to dengue but unlike most other arboviral infections). ${ }^{7}$

Clinical illness is a typical viral syndrome associated with a debilitating symmetrical peripheral arthropathy that lasts weeks to months. Neurological involvement is reported in $15-25 \%$ of cases, ${ }^{11}$ and is commoner in older children and older adults. ${ }^{6,10}$ The spectrum includes meningitis, encephalitis, encephalopathy, encephalomyelitis, GBS, myelopathy, peripheral neuropathy and myopathy. ${ }^{7,10,11}$ Diagnosis is based on detection of serum IgM and IgG antibodies, and viral RNA by RT-PCR in CSF or serum. MRI, EEG and CSF changes are variable. ${ }^{7,10}$ Encephalitis in neonates due to vertical transmission is reported. ${ }^{7,10,11}$

\section{Zika and neurological involvement}

Zika virus (ZIKV, family Flaviviridae, genus Flavivirus, transmitted by Aedes mosquitoes) was first identified in 1947 in a Rhesus monkey in the Zika forest in Uganda. ${ }^{12}$ Neurological complications were first reported in the explosive outbreaks in the Pacific region (2013-2014) and the Americas (2015-2016). 12,13 They include
GBS, encephalitis, encephalopathy, ADEM, myelitis and stroke due to cerebral vasculopathy. ${ }^{12-14} \mathrm{GBS}$ is the commonest neurological manifestation, and is characterised by prominent cranial nerve involvement and autonomic dysfunction, short latency period following ZIKV infection, and rapid progression. ZIKV-associated encephalitis presents with concomitant neuromuscular weakness, similar to other flaviviral encephalitides. MRI changes are seen in subcortical areas, and CSF shows a neutrophilic pleocytosis. Detection of RNA (by RT-PCR) and IgM in CSF helps establish the association with ZIKV infection. ${ }^{12-14}$

An epidemic of microcephaly in Brazil, with a 17-fold increase in numbers during the 2014-2015 outbreak, led to the recognition that ZIKV infection during early pregnancy results in a 'congenital Zika syndrome' (microcephaly, cerebral atrophy, intracranial calcifications, neural tube defects, optic nerve abnormalities and hearing loss). ${ }^{12,14,15}$ ZIKV genomes (by RT-PCR) and IgM antibodies were detected in the CSF and serum of newborns, and ZIKV-RNA in amniotic fluid and placentae of pregnant mothers and foetal brain tissue of dead infants. ${ }^{14,15}$

\section{Other emerging and re-emerging infections}

Several other emerging and re-emerging infections with neurological involvement have received increasing global attention.

$>$ Chandipura virus was responsible for recent outbreaks of acute encephalitis in children associated with high case fatality (55-78\%) in central India. It is considered to contribute significantly to the case load of acute encephalitis in the country. ${ }^{1,16}$

> Melioidosis, caused by Burkholderia pseudomallei, a facultative intracellular bacterium, is prevalent in South and Southeast Asia and Northern Australia. Neurological melioidosis is increasingly being reported, and can present as cerebral and epidural abscesses, brainstem encephalitis, aseptic meningitis, transverse myelitis and dural venous thrombosis. ${ }^{17}$

> Ebola virus disease is a viral haemorrhagic fever, and neurological manifestations came into prominence during the 2014-2016 outbreak in West Africa. ${ }^{18}$ They include seizures, encephalopathy, encephalitis, meningitis and frontal lobe dysfunction. It is believed that the virus can lie dormant in immunologically privileged sanctuary sites such as the CNS, and produce late relapsing infection and delayed transmission. ${ }^{18,19}$

These infections are at present confined to their geographical regions, but their potential for transcontinental spread due to international air travel must be recognised.

\section{Conclusions}

Up to $85 \%$ of neurological infections worldwide are due to undetected causes, ${ }^{20}$ and emerging infections are likely to be responsible for a fair proportion of these. We need to start searching beyond the traditional diagnostic algorithms for hitherto unsuspected pathogens.

Emerging pathogens may produce regional or global outbreaks by expanding their geographical range (eg Chiukungunya), spreading from animal reservoirs (eg Nipahvirus), or acquiring new neurovirulence (eg Chiukungunya). ${ }^{6,7}$ The pandemic potential of these emerging infections (eg Nipahvirus) ${ }^{8}$ becomes increasingly relevant in the light of the ongoing COVID-19 pandemic. The health of the humans, animals and the planet are closely 
intertwined, and the recognition of this 'One Health' concept in disease epidemiology would help us be better prepared when the next pandemic comes calling.

\section{References}

1 Joshi R, Kalantri SP, Reingold A, Colford JM. Changing landscape of acute encephalitis syndrome in India: a systematic review. Nat/ Med J India 2012;25:212-20.

2 Ranawaka UK. The challenge of treating central nervous system infections in the developing world. J Ceylon Coll Physicians 2018:49:2-15.

3 World Health Organisation Fact Sheets. Dengue and severe dengue. www.who.int/news-room/fact-sheets/detail/dengue-andsevere-dengue [Accessed 12 December 2021].

4 Carod-Artal F], Wichmann O, Farrar ], Gascón J. Neurological complications of dengue virus infection. Lancet Neurol 2013; 12:906-19.

5 Puccioni-Sohler M, Rosadas C, Cabral-Castro MJ. Neurological complications in dengue infection: a review for clinical practice. Arq Neuropsiquiatr 2013;71:667-71.

6 Griffin DE. Emergence and re-emergence of viral diseases of the central nervous system. Prog Neurobiol 2010;91:95-101.

7 Tyler KL. Emerging viral infections of the central nervous system: Part 1. Arch Neurol 2009;66:939-48.

8 Hauser N, Gushiken AC, Narayanan S, Kottilil S, Chua J V. Evolution of nipah virus infection: past, present, and future considerations. Trop Med Infect Dis 2021;6:24

9 Ong KC, Wong KT. Henipavirus encephalitis: recent developments and advances. Brain Pathol 2015;25:605-13.

10 Arpino C, Curatolo P, Rezza G. Chikungunya and the nervous system: what we do and do not know. Rev Med Virol 2009;19:121-9.

11 Das T, Jaffar-Bandjee MC, Hoarau J] et al. Chikungunya fever: CNS infection and pathologies of a re-emerging arbovirus. Prog Neurobiol 2010;91:121-9.
12 Tyler KL, Roos KL. The expanding spectrum of Zika virus infections of the nervous system. JAMA Neurol 2017;74:1169-71.

13 Da Silva IRF, Frontera JA, De Filippis AMB, Do Nascimento OJM. Neurologic complications associated with the Zika virus in Brazilian adults. JAMA Neurol 2017;74:1190-8.

14 Carod-Artal FJ. Neurological complications of Zika virus infection. Expert Rev Anti Infect Ther 2018;16:399-410.

15 de Araújo TVB, Ximenes RA de A, Miranda-Filho D de B et al. Association between microcephaly, Zika virus infection, and other risk factors in Brazil: final report of a case-control study. Lancet Infect Dis 2018;18:328-36.

16 Rao BL, Basu A, Wairagkar NS et al. A large outbreak of acute encephalitis with high fatality rate in children in Andhra Pradesh, India, in 2003, associated with Chandipura virus. Lancet 2004:364:869-74.

17 Fong SL, Shyanwong J, Tan AH, Low SC, Tan CT. Neurological melioidosis in East Malaysia: Case series and review of the literature. Neurol Asia 2017:22:25-32

18 Mailles A, Stahl JP, Bloch KC. Update and new insights in encephalitis. Clin Microbiol Infect 2017;23:607-13.

19 Malvy D, McElroy AK, de Clerck H, Günther S, van Griensven J. Ebola virus disease. Lancet 2019;393:936-48.

20 Granerod J, Ambrose HE, Davies NWS et al. Causes of encephalitis and differences in their clinical presentations in England: a multicentre, population-based prospective study. Lancet Infect Dis 2010;10:835-44.

Address for correspondence: Prof Udaya K Ranawaka, Department of Medicine, Faculty of Medicine, University of Kelaniya, PO Box 6, Ragama, Sri Lanka.

Email: udayaran@kln.ac.lk 